\title{
2.15 PHOTOMETRY AND POLARIMETRY OF THE CRAB PULSAR
}

\author{
N. VISVANATHAN \\ Harvard College Observatory, Cambridge, Mass., U.S.A.
}

\begin{abstract}
Observational results are presented for the pulse component, the nebula component and the wisp component of the Crab Nebula. The pulsar polarization is separated from the background nebular polarization. The relation between the pulsar and the adjacent nebula is discussed.
\end{abstract}

1. The discovery that the central star of the Crab Nebula pulsates with the shortest period known, $33 \mathrm{msec}$, played a decisive role to the idea put forward by Gold, that pulsars are rotating neutron stars. Scargle (1969) has studied the semi-periodic nature of wisps around the pulsar and recently he and Harlan (1970) found evidence that the recent spin up of the pulsar in September 1969 was followed by a change of structure in the wisps. Further Arecibo observations (reported in this Conference) show an increase in the dispersion measure in the direction of the Crab. All these show an intimate electrodynamic link between the Crab Nebula and pulsar in the center.

In this paper, observational results obtained for the pulse component, nebula component and wisp component are presented. These observations were made at the prime focus of 200-in. telescope in collaboration with Dr. Kristian. For a description of instrumentation used, kindly refer to Kristian et al. (1970). Nebula observations were made at the Cassegrain focus of 84-inch at Kitt Peak National Observatory with an automatic photometer-polarimeter.

Figure 1 is the $50 \mu \mathrm{sec}$ light curve of the pulsar obtained on 18 October 1969 with a total integration time of $1 \mathrm{~h}$. The following features are to be noted in the figure:

1. (a) The asymmetrical shape of the main and sub pulses with respect to the peak. The falling edge is steeper than the rising edge in the case of the main pulse while it is the other way around in the case of sub-pulse.

(b) The peak is sharp and the intensity reaches peak within an interval of time $40 \mu \mathrm{sec}$ and the width at $90 \%$ of maximum is of the order of $100 \mu \mathrm{sec}$. This means that the core of the pulse, if it is a sweeping beam, has an angular size of a degree.

(c) In between the main and sub pulses the intensity is more than that in the other side of the pulse. This excess is about $1.5 \%$ of the peak intensity of the main pulse. This implies that the main pulse has a long tail.

(d) The peaks of the main and sub pulses are separated by $13.5 \mathrm{msec}$ which is 0.41 of the phase.

(e) The area of the main pulse is twice that of the sub pulse.

2. Figure 2 is a comparison of the light curve of the pulsar in two filters - visual $(5500 \AA)$ and ultraviolet $(3600 \AA)$. Each point in the pulse corresponds to $40 \mu \mathrm{sec}$. To make the comparison easier the intensities are plotted on a log scale and shifted vertically. It can be seen that the differences between two filters at different points in the phase is about a few percent. Thus the spectrum at different points at the pulse is exactly the same within a few percent. 


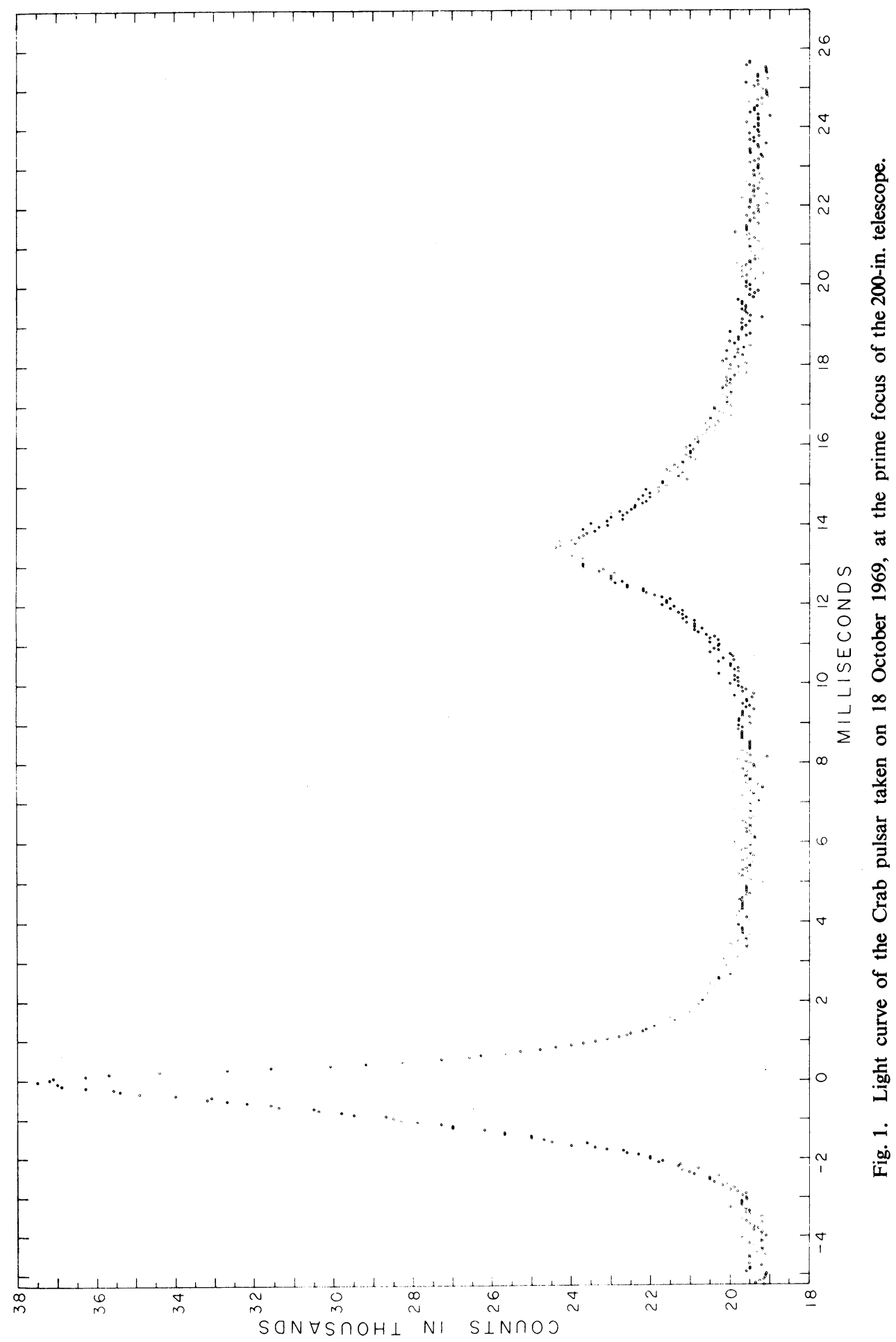


3. The linear polarization of the pulsar was measured on the nights of 8 February, 18 and 19 August, 19 October and 3 December 1969. Most of the polarization measurements were made by rotating the polaroid filter in $30^{\circ}$ steps from $0^{\circ}$ to $360^{\circ}$. The starting time of each run was also known to within 50 microsec so that the detailed time behaviour of the polarization within the averaged pulses could be studied.

4. Figure 3 is the plot of $\mathbf{P}$ and $\boldsymbol{\theta}$ versus phase of the pulse. Each point corresponds to the integrated $\mathbf{P}$ and $\boldsymbol{\theta}$ for $\mathbf{4}$ channels. It can be seen that $\mathbf{P}$ and $\boldsymbol{\theta}$ are different only where the pulses occur, otherwise they are constant. The constant polarization represents the polarization of nebular light around the pulsar. The polarization at each point in the pulse represents the vectorial addition of the constant nebular polarization and the polarization of the pulse at that point. The fact that $\mathbf{P}$ and $\boldsymbol{\theta}$ of the

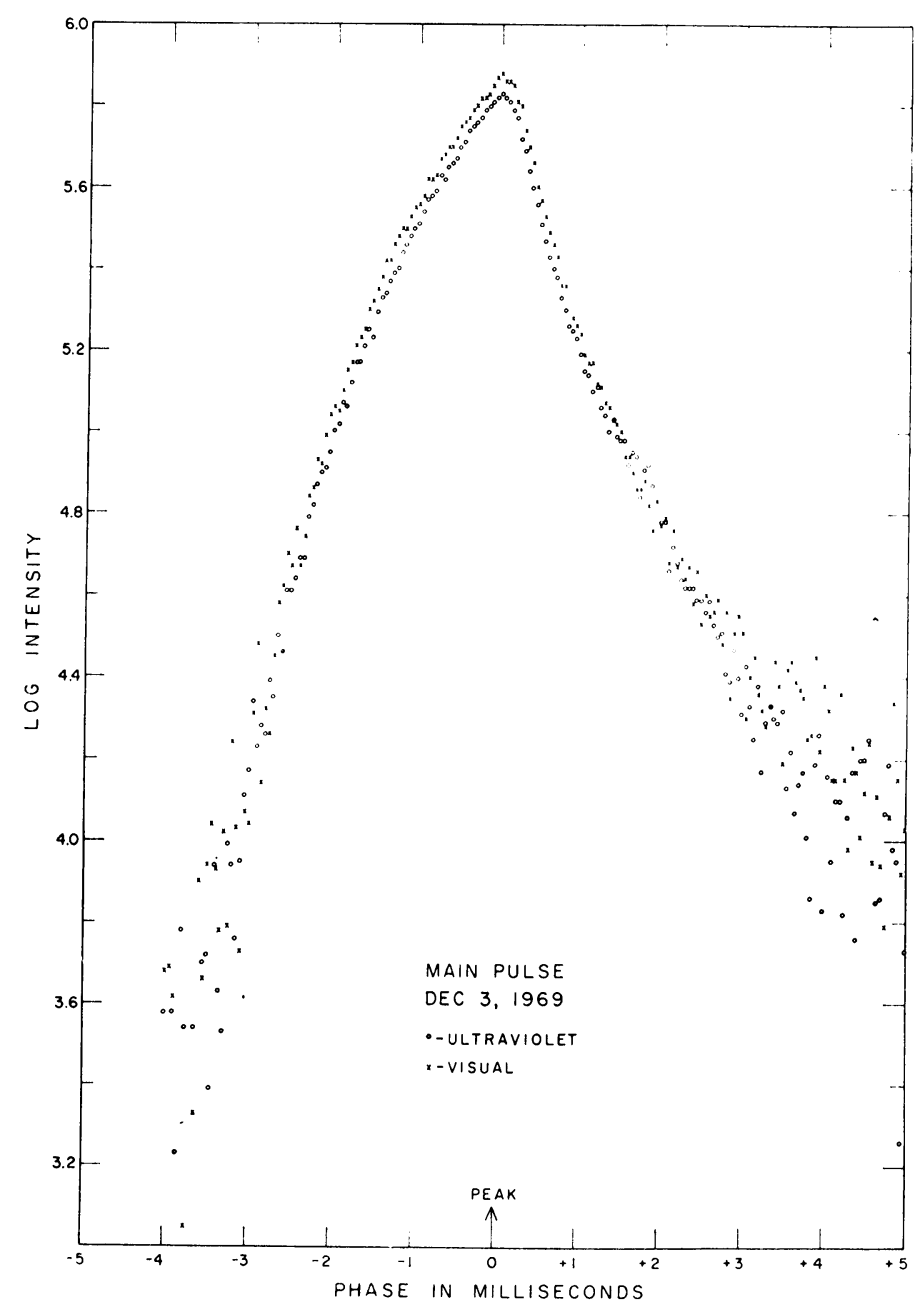

Fig. 2. Comparison of main pulse at visual and ultraviolet wavelengths. 
pulses are different from nebular polarization indicates the pulses are linearly polarized.

2. After subtracting the background polarization at each point, the runs of $\mathbf{P}$ and $\boldsymbol{\theta}$ within the main and sub pulses have been constructed. The results in both the pulses show that $\mathbf{P}$ drops smoothly from about $25 \%$ in the leading edge to a minimum of about two percent just after the peak of the light curve, then increases in a roughly symmetric fashion to at least $10 \%$ in the trailing edge. The position angle of the electric vector $\boldsymbol{\theta}$ varies continuously from the rising edge of the pulse to the falling side of the pulse from $80^{\circ}$ to $50^{\circ}$. The rotation of the electric vector within the pulses can be explained by a general geometrical model by assuming that the pulses are due to a

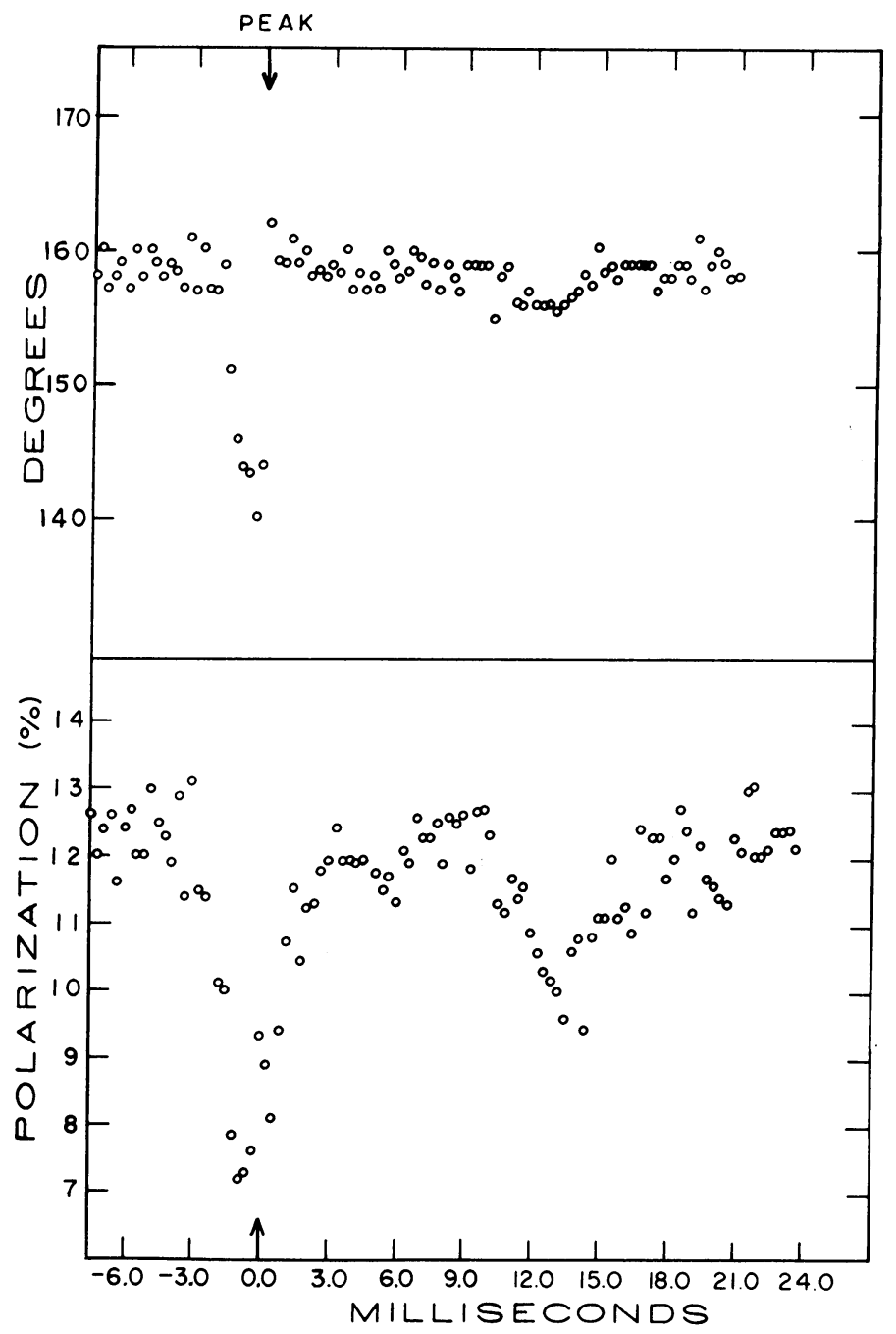

Fig. 3. Run of $\mathbf{P}$ and $\boldsymbol{\theta}$ with phase of the Crab pulsar along with background nebula, observed with $7 \mathrm{sec}$ of arc aperture, at the prime focus of the 200-in. telescope, on 18 October 1969. 
polarized radiation pattern fixed in the pulsar which is scanned as the pulsar rotates For each of the pulses we assume a unit vector $p$ fixed in and rotating with pulsar. At any instant, the position angle of electric vector $\boldsymbol{\theta}$ we measure is the angle which the projection of the vector $\mathbf{P}$ makes in the plane of the sky. The observed run of $\boldsymbol{\theta}$ with phase suggests that the observer must lie within $35^{\circ}$ of the equator and the emission pattern in the pulsar must lie within $25^{\circ}$ of the equator.

Another feature of this model is the run of $\boldsymbol{\theta}$ with phase and it will show inversion symmetry about $\omega t=0$ (when the observer is at the longitude of the emission pattern) when the vector $\mathbf{P}$ lies in the plane defined by rotation axis and the observer. At this time the electric vector $\boldsymbol{\theta}$ will be either parallel or perpendicular to the projection of the rotation axis on the plane of the sky. This angle comes out to be $160^{\circ}$. It is interesting to note that the nebular light very near to the pulsar is polarized and the position angle of the electric vector $\theta$ is $160^{\circ}$. These show that the magnetic field around the pulsar is either parallel or perpendicular to the rotation axis of the pulsar. We think that this relationship between rotation axis and the outside field suggests a direct causal connection between the pulsar and the nebula.

Linear polarization observations of the nebula were made with different apertures ranging from $2 \mathrm{sec}$ of arc to $20 \mathrm{sec}$ of arc around the pulsar.

These observations show a region of constant $\mathbf{P}$ and $\boldsymbol{\theta}$ asymmetrically situated with respect to the pulsar, extending $16 \mathrm{sec}$ in the west and $8 \mathrm{sec}$ in the east. These indicate that the magnetic field structure around the pulsar is uniform in that region and the fine scale structure is smaller than 0.02 parsec. The uniform field in this region is at an angle of $69^{\circ}$. In the northwest side of the pulsar there are many wisps seen in the continuum photographs. The axes of these wisps are at an angle of $30^{\circ}$ to the general magnetic field in this region. Scargle and Harlan (1970) found that these wisps had changed their structure after the September jump in the period of the pulsar, thus suggesting a relation between the two phenomena. There is an indication that the magnetic fields in the wisps are at an angle $30^{\circ}$ from the general magnetic field around the pulsar.

Thus the Crab nebula shows a linearly polarized pulse component within which electric vector $\boldsymbol{\theta}$ rotates, surrounded by a constantly polarized nebular component and slowly variable polarized wisp component.

\section{References}

Kristian, J., Visvanathan, N., Westphal, J. A., and Snellen, G. H.: 1970, Astrophys. J. 162 , 475.

Scargle, J. D.: 1969, Astrophys. J. 156, 401.

Scargle, J. D. and Harlan, E.: 1970, Astrophys. J. Letters 159, L143. 